\title{
ON THE ERGODIC HILBERT TRANSFORM FOR LAMPERTI OPERATORS
}

\author{
RYOTARO SATO
}

\begin{abstract}
This paper is devoted to the proof of almost everywhere existence of the ergodic Hilbert transform for a class of Lamperti operators.
\end{abstract}

1. Introduction. Let $(X, \mathcal{F}, \mu)$ be a $\sigma$-finite measure space and $T$ a bounded linear operator on $L_{p}=L_{p}(X, \mathcal{F}, \mu), 1 \leq p<\infty$. T is called a Lamperti operator if it maps functions with disjoint support to the same. It is known (see e.g. $[\mathbf{7}, \mathbf{9}]$ ) that Lamperti operators include $L_{p}$ isometries, $p \neq 2$, and positive $L_{2}$ isometries. If $T$ is invertible, then by considering known results about the classical discrete Hilbert transform, the following question arises: Does the limit

$$
H f=\lim _{n \rightarrow \infty} \sum_{k=-n}^{n} \frac{1}{k} T^{k} f \quad\left(f \in L_{p}\right)
$$

exist in any sense? (Here the prime means that the term with zero denominator is omitted.) Under the assumption that $T$ is induced by an invertible measure preserving transformation, Cotlar $[3]$ proved that if $1<p<\infty$, then (1) exists almost everywhere and in the strong operator topology; if $p=1$, then (1) exists almost everywhere (see also Calderón [2] and Petersen [10]). In this paper we shall assume that $T$ is an invertible Lamperti operator such that $\sup \left\{\left\|T^{n}\right\|_{p}:-\infty<n<\right.$ $\infty\}<\infty$, and prove that if $1<p<\infty$, then (1) exists almost everywhere and in the strong operator toplology; if $p=1$, then, under the additional hypothesis that $\sup \left\{\left\|T^{n}\right\|_{\infty}:-\infty<n<\infty\right\}<\infty,(1)$ exists almost everywhere. It is interesting to note that the author proved in $[\mathbf{1 1}]$ that if $T$ is an invertible positive operator on $L_{p}, 1<p<\infty$, such that $\sup \left\{\left\|T^{n}\right\|_{p}:-\infty<n<\infty\right\}<\infty$ then (1) exists almost everywhere and in the strong operator topology.

2. Results. The following maximal theorem is a key lemma to prove the almost everywhere existence of the ergodic Hilbert transform.

THEOREM 1. Let $T$ be an invertible Lamperti operator on $L_{p}, 1<p<\infty$, such that $\sup \left\{\left\|T^{n}\right\|_{p}:-\infty<n<\infty\right\}=M<\infty$. Define the ergodic maximal Hilbert transform $H^{*}$, associated with $T$, as

$$
H^{*} f=\sup _{n \geq 1}\left|\sum_{k=-n}^{n} \frac{1}{k} T^{k} f\right| .
$$

Received by the editors September 6, 1985 .

1980 Mathematics Subject Classification (1985 Revision). Primary 47A35.

Key words and phrases. Ergodic Hilbert transform, Lamperti operators, $L_{p}$ isometries, measure preserving transformations. 
Then there exists a constant $C>0$, depending only on $M$, such that $\left\|H^{*} f\right\|_{p} \leq$ $C\|f\|_{p}$ for all $f \in L_{p}$.

Proof. It follows from Lamperti [9] (cf. also Kan [8]) that there exists a $\sigma$ endomorphism $\Phi$ of the Boolean $\sigma$-algebra $\mathcal{F}(\mu)$, associated with the measure space $(X, \mathcal{F}, \mu)$, and a measurable function $h$ on $X$ such that

$$
T f=h \cdot \Phi f \quad \text { for all } f \in L_{p},
$$

where we denote by the same letter $\Phi$ the linear operator on the space of measurable functions induced by the $\sigma$-endomorphism. Since $T$ is invertible by hypothesis, $|h|>0$ on $X$ and $\Phi$ is one-to-one and onto from $\mathcal{F}(\mu)$ to $\mathcal{F}(\mu)$. Thus we can define

$$
U f=\frac{1}{\Phi^{-1} h} \Phi^{-1} f \quad\left(f \in L_{p}\right) .
$$

Then

$$
U T f=\frac{1}{\Phi^{-1} h} \Phi^{-1}(T f)=\frac{1}{\Phi^{-1} h}\left(\Phi^{-1} h\right) f=f,
$$

and so $U=T^{-1}$. Put $h_{1}=h, h_{0}=1, h_{-1}=1 / \Phi^{-1} h, h_{n}=h_{1} \cdot \Phi h_{n-1}$, and $h_{-n}=h_{-1} \cdot \Phi^{-1} h_{-n+1}(n \geq 2)$. It follows that

$$
T^{j} f=h_{j} \cdot \Phi^{j} f \quad(j=0, \pm 1, \ldots)
$$

and

$$
h_{j+k}=h_{j} \cdot \Phi^{j} h_{k} \quad(j, k=0, \pm 1, \ldots) ;
$$

in fact (4) follows from the equalities

$$
h_{j+k} \cdot \Phi^{j+k} f=T^{j+k} f=T^{j}\left(h_{k} \Phi^{k} f\right)=h_{j} \cdot \Phi^{j} h_{k} \cdot \Phi^{j+k} f .
$$

For an integer $K \geq 1$, define the truncated maximal operator $H_{K}^{*}$ as

$$
H_{K}^{*} f=\max _{1 \leq n \leq K}\left|\sum_{k=-n}^{n} \frac{1}{k} T^{k} f\right| \quad\left(=\max _{1 \leq n \leq K}\left|\sum_{k=-n}^{n} \frac{1}{k} h_{k} \cdot \Phi^{k} f\right|\right) .
$$

Since $\left\|T^{j}\right\|_{p} \leq M$ for all $j$, it then follows that

$$
\begin{aligned}
\left\|H_{K}^{*} f\right\|_{p}^{p} & \leq \frac{M^{p}}{2 L+1} \int \sum_{j=-L}^{L}\left|T^{j} H_{K}^{*} f\right|^{p} d \mu \\
& =\frac{M^{p}}{2 L+1} \int \sum_{j=-L}^{L}\left|h_{j} \cdot \Phi^{j}\left(H_{K}^{*} f\right)\right|^{p} d \mu \\
& =\frac{M^{p}}{2 L+1} \int \sum_{j=-L}^{L}\left|h_{j}\left[\max _{1 \leq n \leq K}\left|\sum_{k=-n}^{n} \frac{1}{k} \Phi^{j} h_{k} \cdot \Phi^{j+k} f\right|\right]\right|^{p} d \mu \\
& =\frac{M^{p}}{2 L+1} \int \sum_{j=-L}^{L}\left[\max _{1 \leq n \leq K}\left|\sum_{k=-n}^{n} \frac{1}{k} h_{j+k} \cdot \Phi^{j+k} f\right|\right]^{p} d \mu \\
& \leq \frac{M^{p}}{2 L+1} C^{p} \sum_{j=-L-K}^{L+K}\left|h_{j} \cdot \Phi^{j} f\right|^{p} d \mu \\
& \leq \frac{M^{p}}{2 L+1} C^{p} 2(L+K+1) M^{p}\|f\|_{p}^{p},
\end{aligned}
$$


where the second inequality from the bottom is due to a known result about the classical discrete Hilbert transform (see e.g. Hunt, Muckenhoupt, and Wheeden [6]); letting $L \rightarrow \infty$, we get

$$
\left\|H_{K}^{*} f\right\|_{p}^{p} \leq M^{2 p} C^{p}\|f\|_{p}^{p} .
$$

This completes the proof, since $H_{K}^{*} f \uparrow H^{*} f$.

THEOREM 2. Let $T$ be as in Theorem 1. Then the limit (1) exists almost everywhere and in the strong operator toplology.

PROOF. See the proof of Theorem 2 in $[\mathbf{1 1}]$.

THEOREM 3. Let $T$ be an invertible Lamperti operator on $L_{1}$ such that

$$
\sup \left\{\left\|T^{n}\right\|_{1}:-\infty<n<\infty\right\}=M_{1}<\infty
$$

and

$$
\sup \left\{\left\|T^{n}\right\|_{\infty}:-\infty<n<\infty\right\}=M_{\infty}<\infty
$$

Then the limit (1) exists almost everywhere.

To prove this, we need the following lemma.

LEMMA. Let $T$ be as in Theorem 3. Then there exists a constant $C>0$ such that for any $f \in L_{1}$ and $\varepsilon>0$

$$
\mu\left\{H^{*} f>\varepsilon\right\} \leq \frac{c}{\varepsilon}\|f\|_{1} .
$$

ProOF. Since $T^{j} f=h_{j} \cdot \Phi^{j} f$ by (3), it may be assumed without loss of generality that $\mathcal{F}$ is generated by a countable collection of sets in $X$. Then, by considering a finite equivalent measure and making use of the isomorphism of a separable nonatomic normalized measure algebra with the measure algebra of the unit interval (cf. [5, p. 173]), we observe that $\mathcal{F}(\mu)$ is $\sigma$-isomorphic to the Boolean $\sigma$-algebra $B(\lambda)$ associated with a certain measure space $([0,1], B, \lambda)$, where $B$ is the Borel subset of $[0,1]$ and $\lambda$ is a finite measure. Thus we may assume that $X=[0,1]$ and $\mathcal{F}(\mu)=B(\lambda)$. Since $\Phi$ is one-to-one and onto from $\mathcal{F}(\mu)$ to $\mathcal{F}(\mu)$, it follows (see e.g. $[1$, pp. $69-73])$ that there exists a one-to-one and onto mapping $S$ from $X$ to $X$ such that

(i) $A \in B$ if and only if $S A \in B$,

(ii) $\lambda A>0$ if and only if $\lambda(S A)>0$,

(iii) for each integer $j$ and measurable function $f, \Phi^{j} f=f \circ S^{j}$.

It follows from (4) that for almost all $x \in X$

$$
h_{j+k}(x)=h_{j}(x) h_{k}\left(S^{j} x\right) \quad(j, k=0, \pm 1, \ldots) .
$$

Therefore for almost all $x \in X$

$$
\begin{aligned}
\left|\left(T^{j} H_{K}^{*}\right)(x)\right| & =\left|h_{j}(x) H_{K}^{*} f\left(S^{j} x\right)\right| \\
& =\max _{1 \leq n \leq K}\left|\sum_{k=-n}^{n} \frac{1}{k} h_{j}(x) h_{k}\left(S^{j} x\right) f\left(S^{j+k} x\right)\right| \\
& =\max _{1 \leq n \leq K}\left|\sum_{k=-n}^{n} \frac{1}{k} h_{j+k}(x) f\left(S^{j+k} x\right)\right| .
\end{aligned}
$$


From this and the fact that $\left\|T^{-j}\right\|_{\infty}=\left\|1 / h_{j}\right\|_{\infty} \leq M_{\infty}$ it follows that

$$
H_{K}^{*} f\left(S^{j} x\right) \leq M_{\infty} \max _{1 \leq n \leq K}\left|\sum_{k=-n}^{n} \frac{1}{k} h_{j+k}(x) f\left(S^{j+k} x\right)\right| .
$$

Since $\left\|T^{j}\right\|_{1} \leq M_{1}$ and $\left\|T^{j}\right\|_{\infty}=\left\|h_{j}\right\|_{\infty} \leq M_{\infty}$ for all $j$, we then have

$$
\begin{aligned}
& (2 L+1) \mu\left\{H_{K}^{*} f>\varepsilon\right\} \leq M_{1} \sum_{j=-L}^{L}\left\|T^{j} \chi_{\left\{H_{K}^{*} f>\varepsilon\right\}}\right\|_{1} \\
& \quad=M_{1} \int \sum_{j=-L}^{L}\left|h_{j}(x)\right| \chi_{\left\{H_{K}^{*} f>\varepsilon\right\}}\left(S^{j} x\right) d \mu(x) \\
& \quad=M_{1} \int_{\left\{j:-L \leq j \leq L, H_{K}^{*} f\left(S^{j} x\right)>\varepsilon\right\}}\left|h_{j}(x)\right| d \mu(x) \\
& \quad \leq M_{1} \sum_{\left\{j:-L \leq j \leq L, \varepsilon / M_{\infty}<\max _{1 \leq n \leq K}\left|\sum_{k=-n}^{\prime n}(1 / k) h_{j+k}(x) f\left(S^{j+k} x\right)\right|\right\}}\left|h_{j}(x)\right| d \mu(x) \\
& \quad \leq \frac{M_{1} M_{\infty}^{2}}{\varepsilon} \int \sum_{j=-L-K}^{L+K}\left|h_{j}(x) f\left(S^{j} x\right)\right| d \mu(x) \\
& \quad \leq \frac{M_{1} M_{\infty}^{2}}{\varepsilon} C 2(L+K+1) M_{1}\|f\|_{1},
\end{aligned}
$$

where the second inequality from the bottom is due to a known result about the classical discrete Hilbert transform (see e.g. [6]). Letting $L \rightarrow \infty$, we get

$$
\mu\left\{H_{K}^{*} f>\varepsilon\right\} \leq \frac{M_{1}^{2} M_{\infty}^{2}}{\varepsilon} C\|f\|_{1}
$$

and the proof is completed.

PROOF OF THEOREM 3. The Riesz convexity theorem implies $\left\|T^{n}\right\|_{p} \leq$ $\max \left\{M_{1}, M_{\infty}\right\}$ for all integers $n$ and $p, 1<p<\infty$. Thus, by Theorem 2 , the limit (1) exists almost eveywhere for all $f \in L_{1} \cap L_{p}$. Since $H^{*} f<\infty$ almost everywhere for all $f \in L_{1}$ by the above lemma, and since $L_{1} \cap L_{p}$ is a dense subspace of $L_{1}$, Banach's convergence theorem (see e.g. [4, p. 332]) establishes Theorem 3.

\section{REFERENCES}

1. P. Billingsley, Engodic theory and information, Wiley, New York, 1965.

2. A. P. Calderón, Ergodic theory and translation-invariant operators, Proc. Nat. Acad. Sci. U.S.A. 59 (1968), 349-353.

3. M. Cotlar, A unified theory of Hilbert transforms and ergodic theorems, Rev. Mat. Cuyana 1 (1955), 105-167.

4. N. Dunford and J. T. Schwartz, Linear operators. I: General theory, Interscience, New York, 1958.

5. P. R. Halmos, Measure theory, Van Nostrand, New York, 1950.

6. R. Hunt, B. Muckenhoupt, and R. Wheeden, Weighted norm inequalities for the conjugate function and Hilbert transform, Trans. Amer. Math. Soc. 176 (1973), 227-251.

7. A. Ionescu Tulcea, Ergodic properties of isometries in $L^{p}$ spaces, $1<p<\infty$, Bull. Amer. Math. Soc. 70 (1964), 366-371. 
8. C. H. Kan, Ergodic properties of Lamperti operators, Canad. J. Math. 30 (1978), 1206-1214.

9. J. Lamperti, On the isometries of certain function-spaces, Pacific J. Math. 8 (1958), 459-466.

10. K. Petersen, Another proof of the existence of the ergodic Hilbert transform, Proc. Amer. Math. Soc. 88 (1983), 39-43.

11. R. Sato, On the ergodic Hilbert transform for operators in $L_{p}, 1<p<\infty$ (submitted).

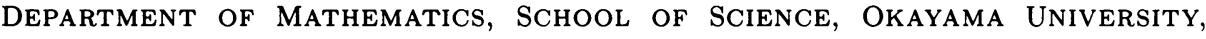
OKAYAMA, 700, JAPAN 